\title{
Consumo alimentar e os pressupostos dos tipos de estilos de vida atual
}

O consumo alimentar nas sociedades atuais se tornou um dos principais aspectos para as vidas dos indivíduos sociais, esse dita os padrões alimentares diferentes em todo o globo, entretanto, são perceptíveis categorias e subcategorias de tipos de consumos alimentares, influenciado pela globalização e a produção em massa dos alimentos. Neste sentido, o trabalho cria, de forma inédita, categorias especificas para os determinados padrões dos consumos alimentares, a partir dos referenciais de todo o globo, divulgados pelos principais órgãos de controle e de segurança mundial da produção e do consumo dos alimentos. Os padrões alimentares são: 1) 'seguidores das dietas sustentáveis'; 2) 'seguidores do consumo alimentar industrializado e urbanizado'; 3) 'os seguidores de dietas da suficiência'; 4) 'sem entendimento de sua alimentação'; 5) 'criativos e inovadores'. Com o método hipotético-dedutivo se analisam os tipos dos estilos de vida, de forma global e transversal, que surgem através do mercado globalizado de consumo dos alimentos. Percebe-se que as influências das principais políticas do controle e da produção de alimentos moldam e são moldados pelo mercado de consumo de alimentos. Os indivíduos sociais são identificados, muitas vezes, pelos seus tipos de consumo alimentares, estes são os motivos iniciais e finais de toda a produção em massa de alimentos e as inovações dos seus variados segmentos.

Palavras-chave: Padrões alimentares; Consumo Alimentar; Produção de Alimentos; Indivíduos sociais.

\section{Food consumption and the assumptions of types of current lifestyles}

Food consumption in today's societies has become one of the main aspects for the lives of social individuals, this dictates the different eating patterns across the globe, however, there are perceptible categories and subcategories of types of food consumption, influenced by globalization and production in mass of food. In this sense, the work creates, in an unprecedented way, specific categories for the certain patterns of food consumption, based on references from all over the globe, released by the main organs of control and world security of food production and consumption. The eating patterns are: 1 ) 'followers of sustainable diets'; 2) 'followers of industrialized and urbanized food consumption'; 3) 'followers of sufficiency diets'; 4) 'without understanding your food'; 5) 'creative and innovative'. With the hypothetical-deductive method, the types of lifestyles are analyzed, in a global and transversal way, that arise through the globalized food consumption market. It is perceived that the influences of the main policies of food control and production shape and are shaped by the food consumption market. Social individuals are often identified by their types of food consumption, these are the initial and final reasons for all mass production of food and the innovations of their various segments.

Keywords: Food standards; Food Consumption; Food production; Social individuals.

Topic: Ciências Humanas e Sociais em Alimentação e Nutrição

Reviewed anonymously in the process of blind peer
Received: $20 / 04 / 2019$

Approved: 14/08/2019

Rosilene Gonçalves de Oliveira

Universidade Estácio de Sá, Brasi

http://lattes.cnpq.br/2505475681076691

rosilene nutricionista@outlook.com

Naccer Cayc Ribeiro Donato (iD

Centro Universitário Internacional, Brasil

http://lattes.cnpq.br/4285077522771135

http://orcid.org/0000-0001-8162-1009

naccercaycrd@gmail.com

Referencing this:

OLIVEIRA, R. G.; DONATO, N. C. R.. Consumo alimentar e os pressupostos dos tipos de estilos de vida atual. Health of Humans, v.1 n.2, p.8-17, 2019. DOI: http://doi.org/10.6008/CBPC26746506.2019.002.0002 


\section{INTRODUÇÃO}

Hoje, os problemas associados ao consumo alimentares indevido crescem de uma forma acelerada; as gorduras corporais em excesso no ser humano demonstram uma série de riscos à saúde, já a obesidade é um problema social mundial. Então, surge uma série de estilos de vida ligados diretamente ao consumo alimentar, muitos associados a determinadas ideologias, que estão se configurando de várias maneiras no senso comum. Os estilos de vidas alimentares representam ora soluções para prevenções de problemas de saúde e ora riscos eminentes a vida humana relativamente saudável. Segundo Almeida et al. (2009), a comunidade científica realiza vários esforços para reeducar a população para instruir sobre as dietas populares, de forma que corrija suas incoerências e seus riscos a vida.

As várias dietas populares fazem os profissionais de saúde e os pensadores atuais a si perguntarem: que evolução ou adaptação o corpo humano passa na atualidade? Posteriormente, as mudanças de hábitos geram transformações na fisiologia do ser humano? E como o consumo alimentar afeta no futuro da humanidade? Segundo Guerra et al. (2017), os aspectos culturais de uma determinada população estão relacionados diretamente aos seus hábitos alimentares. "A alimentação, além de representar a forma de sustento físico humano, também, fortalece a dignidade das pessoas" (GUERRA et al., 2017).

A partir de artigos e referenciais bibliográficos, publicados nos anos 2000 até o presente, se investiga através de um estudo sistemático de novas pesquisas científicas teóricas e práticas, os tipos dietéticos de estilos de vida que se configuram pela cultural popular na atualidade. $\mathrm{O}$ trabalho visa analisar os hábitos alimentares e o consumo alimentar sobre as alterações socioculturais e fisiológicas dos seres humanos, além de comparar os tipos de dietas populares e sua eficácia no nutrir os indivíduos sociais atuais.

\section{METODOLOGIA}

\section{Estudos teóricos e sistemáticos sobre os padrões alimentares}

As dietas saudáveis mediterrâneas atuais, de acordo com o senso comum, são dietas que se apresentam como umas das melhores opções de consumo alimentar coerente, entretanto todos os aspectos socioculturais mundiais e toda a economia mundial precisa ser considerada e correlacionada com todas as pesquisas para as análises e as comparações sobre os estilos de consumo alimentar, muitas vezes, considerados estilos de vida sustentável.

As dietas mediterrâneas prezam pelas reduções de desperdícios alimentares e da minimização das percas de recursos naturais, isto inclui a água; as novas dietas mediterrâneas se configuram internacionalmente como 'mainframe', que é um conceito de adaptação dos vários estilos de vidas globais às dietas mediterrâneas (CIHEAM et al., 2015).

Os hábitos alimentares mudam constantemente e se transformam de acordo com os avanços tecnológicos da humanidade, segundo pesquisadores brasileiros, Moratoya et al. (2013), identificam os estilos de vidas alimentares urbanos, em geral, como último estágio de mudanças alimentares, aonde o consumo de alimentos mudam os comportamentos dos indivíduos sociais; não se refere apenas ao consumo 
dos padrões econômicos das vidas na globalização, mais aos variados aspectos culturais adquiridos pelos determinados indivíduos sociais.

Os aspectos nutricionais dos consumos de alimentos indicam os vários perfis sociais fundamentados em diferentes tipos de alimentações, segundo Garcia (2003), foi a partir dos anos 90, que os consumos alimentares começaram a se formar como se constrói hoje, no Brasil e no mundo, só entre os anos de 1992 e 1995 as importações de alimentos industrializados no Brasil cresceu 409\%, reflexo de toda globalização que vem se consolidar no século XXI.

As caracterizações das dietas contemporâneas são chamadas, por alguns autores de 'dietas afluentes', que em muitos países são aderidas, reduzem as opções culinárias, aumentam os industrializados; consequentemente, as pessoas de determinados países se acomodam com as alimentações rápidas ('fast foods') e os preparos caseiros das alimentações pelos industrializados pré-prontos (GARCIA, 2003).

Até esta parte do trabalho se identifica dois tipos de estilos de vidas correlacionados aos tipos de consumos de alimentos. Os indivíduos sociais inseguros com a industrialização e a produção em massa da alimentação, que prefere aderir alimentos saudáveis e frescos, que evitem os desperdícios dos recursos naturais, o trabalho chama de 'seguidores das dietas sustentáveis' (em grande parte são adeptos as dietas mediterrâneas mesmo sem saber o conceito desta). Agora, na percepção do aumento dos alimentos industrializados e do crescimento deste mercado, se percebe a maior parte da população mundial aderida as 'dietas afluentes', no trabalho chamados de 'seguidores do consumo alimentar industrializado e urbanizado' (relativo a todos os indivíduos sociais que seguem uma determinada dieta proposta pelo mercado globalizado de alimentos industrializados).

Referente a nutrição e a sua relação com as ciências humanas e sociais, aponta várias incoerências no consumo alimentar, quando procurado profissionais da área da saúde alimentar existe a tentativa de correção e reeducação alimentar, entretanto muitos seguem por si só as dietas populares e os resultados são controversos. Segundo Almeida et al. (2009), as dietas podem ser analisadas pela quantidade de macronutrientes que o indivíduo social ingere, a revisão sistemática dos autores apontam, em 56 dietas avaliadas no Brasil, mais de $50 \%$ da energia é produzido por carboidratos, que relativamente funcionam na dieta como macronutrientes simples que se ingerido em excesso geram gorduras (caso a fonte energética não for utilizada momentaneamente) e em grande parte não são benéfico para o aumento de massa muscular, pois se apresenta como açucares e fontes de energias rápidas para o organismo, que neste caso precisa está em atividade física constante para usar saudavelmente a energia dos carboidratos; também a eficiência das dietas populares são provenientes a redução significativa dos macronutrientes, não significando uma alimentação regular e saudável.

Assim, os profissionais de saúde e os agentes alimentares precisam entender mais os perfis sociais dos indivíduos sociais, no intuito de eliminar as incoerências das dietas modernas atuais, apontam Almeida et al. (2009):

Limitações comuns aos ensaios clínicos de perda de peso incluem falhas na avaliação e manutenção da aderência às dietas propostas e diferenças na intensidade das estratégias adotadas da intervenção. Mais da metade dos estudos incluídos nesta revisão apresentou 
taxa de abandono (perdas de seguimento) a pelo menos um tipo de dieta igual ou maior a $25 \%$. De fato, as taxas de abandono podem ser menores ( $<15 \%)$ em condições diferenciadas como, por exemplo, o fornecimento de refeições em uma mesma área física, no próprio local de trabalho, com cardápio variado e ajustado de acordo com os planos alimentares e com orientações visuais na escolha dos alimentos, além de encontros periódicos com nutricionista específica para cada tipo de dieta (32). (ALMEIDA et al., 2009)

De acordo com as pesquisas, até aqui apresentadas, são várias as complexidades de aderir uma determinada dieta há um determinado estilo de vida. No entanto se aponta: "É certo dizer que a alimentação e o consumo estão intrinsecamente ligados, no que se refere à condição humana de sujeito que precisa dos alimentos como meios de subsistência" (GUERRA et al., 2017). Com as pesquisas apresentadas, o presente trabalho tem como hipótese o surgimento de uma "Cultura de consumo alimentar globalizado", segundo Guerra et al. (2017), existe uma inserção cultural e social das pessoas que aderem determinados tipos de dietas, o trabalho vai além e apresenta de forma transversal uma inserção cultural e social globalizada no mercado mundial de consumo alimentar.

É perceptível que a produção em massa dos alimentos está insustentável no mundo atual, de acordo com Guerra et al. (2017), ao longo da história da modernidade, após a industrialização, há humanidade desenvolveu uma série de hábitos alimentares insustentáveis, sobretudo na produção e no consumo de alimentos de proteína animal, embora pesquisadores tentam implementar novos hábitos que supra a necessidade dos hábitos atuais, afim de corrigir essas incoerências no consumo alimentar. 0 trabalho focará nas ideologias da cultura de consumo e no momento histórico atual, diferente da referência anterior, que foca a história do consumo para apresentar uma crítica as novas tendências alimentares, não discordando dessa, mais para total compreensão desta discussão é preciso analisar quais dietas estão sendo aderidas na globalização e como elas transformam o comportamento humano.

Segundo pesquisadores vinculados a Organização das Nações Unidas (ONU), Moomaw et al. (2012), o período do século XXI é de transição nutricional para a população global, com isto sobre o futuro da produção alimentar é incerto, acontece negligências para atender todos os mercados de consumo alimentar, toda produção global de alimento apresenta $80 \%$ do desmatamento da natureza, representando a maior causa das mudanças climáticas e do uso dos recursos naturais da Terra.

De acordo com os estudos globais sobre o consumo alimentar, Moomaw et al. (2012), o aumento de produção e de consumo de proteína animal é positivo contra o combate à fome, entretanto não ajuda preservar a natureza, com isto a produção alimentar atual é insustentável para o planeta Terra e medidas de conservação aos recursos naturais precisam ser adotadas em grande escala; assim, também, os condutores do mercado de consumo alimentar precisam de alterações para criar novos sistemas de produções e de consumo sustentável.

Na perspectiva dietética, a solução para melhores consumos alimentares, segundo Moomaw et al. (2012), surge o conceito de "sufficiency-based diet" (conjunto de dietas que atendam a bases de nutrientes do corpo humano sem excessos alimentares). No trabalho se configura o terceiro estilo de vida no consumo alimentar, que se chamam 'os seguidores de dietas da suficiência', estes não descaracterizam nenhum tipo de alimento atual, apenas os excessos alimentares. 
Em uma ótica do continente europeu, sobretudo da União Europeia e suas políticas, segundo Maggio et al. (2015), existe uma preocupação com as mudanças climáticas que afetam mundialmente o cultivo de alimentos e isto envolve reeducar uma população para melhor consciência de consumo alimentar; será, cada vez mais, necessárias ações políticas igualitárias que permitam a alimentação de toda população mundial.

A palavra chave para a melhoria do consumo alimentar para os europeus é adaptação, uma nova configuração de preservação dos recursos naturais e uma nova lógica de produção alimentar precisa ser implantada; todas as nações atuais precisam criar políticas convergentes para o tratamento do uso da natureza para a produção de alimentos, considerando os aspectos demográficos e a preservação das biodiversidades restantes dos ecossistemas (MAGGIO et al., 2015).

No referente aos hábitos alimentares e a segurança alimentar global, segundo Maggio et al. (2015), a urbanização gera o aumento intenso dos alimentos derivados do leite, de carnes, sendo processados, que prioriza as alimentações rápidas. Os problemas, segundo essas pesquisas europeias são: as escolhas de todos os tipos de alimentos não são acessíveis para todos; a insegurança alimentar não são preocupações de todos; os alimentos saudáveis se apresentam caros nos supermercados de todo o globo, consequentemente, os necessitados financeiramente, cada vez mais, têm como alimentação os alimentos processados com alto teor de gordura, de açúcar e de sal. O que gera esperança de melhorias são os criativos e os inovadores que movem e transformam todo o mercado global, inclusive os segmentos de produções alimentares; também toda a globalização que reduz a limitações dos desenvolvimentos sociais das zonas urbanas para as zonas rurais (MAGGIO et al., 2015).

Ao refletir sobre esses últimos aspectos socioculturais e sociopolíticos no cenário mundial, se identifica os últimos dois estilos de vida ligados ao consumo alimentar. Primeiramente, os vulneráveis socioeconômicos limitados as suas carências, se chamam 'sem entendimento de sua alimentação', consequentemente, não tem acesso a informação fácil e aos conhecimentos sobre a alimentação, estes vivem conduzidos pelo oferecido e de fácil acessibilidade. O último estilo de vida alimentar, são os 'criativos e inovadores', limitados ou não economicamente, buscam informações e conhecimento sobre o consumo alimentar, como afetam suas vidas e transformam os seus hábitos alimentares em dietas populares adaptados aos seus determinados contextos sociais.

\section{DISCUSSÃO TEÓRICA}

\section{Métodos e reflexões metodológicas da pesquisa}

O trabalho percebe a necessidade de realizar uma análise de estilos de vida que surgem a partir do mercado de consumo de alimentos. O que se realiza é uma pesquisa de referenciais bibliográficos de todo segmento do mercado de consumo de alimentos, focado nos padrões de consumos alimentares e como estes condicionam os estilos de vida da população mundial. O método é hipotético-dedutivo para categorizar os estilos de vida que se apresentam na atualidade, correlacionado com os estudos qualitativos e quantitativos 
das características de consumo alimentar em todo o globo. Assim, Gil (2008) aponta: "Para tentar explicar a dificuldade expressa no problema, são formuladas conjecturas ou hipóteses".

O que o trabalho fórmula é uma inédita conjuntura dos estilos de vida associado ao consumo alimentar, estes são: 1) 'seguidores das dietas sustentáveis'; 2) 'seguidores do consumo alimentar industrializado e urbanizado'; 3) 'os seguidores de dietas da suficiência'; 4) 'sem entendimento de sua alimentação'; 5) 'criativos e inovadores'. Com a hipótese classificada em diferentes conjunturas, surge uma teorização de uma 'Cultura de consumo alimentar globalizado', com conjunturas especificadas de forma original pelos autores embasadas em pesquisas científicas humanas e sociais sobre o consumo alimentar mundial.

Para fundamentação da pesquisa são selecionados sete artigos atuais de revisões científicas literárias e de descrição de dados quanti-qualitativo sobre o consumo alimentar e suas perspectivas de produção e de oferta. Quatro do Brasil para com o mundo, citados nos principais organizadores online de pesquisas acadêmico-científico, exemplos: um agregador de pesquisas científicas da Internet, Scielo; publicações em congressos organizados pelas universidades federais do Brasil. Já os três internacionais são vinculados à órgãos competentes de proteção humana e socioeconômica mundial, são estes: 'European Union' (União Europeia); 'United Nations Environment Programme' (Programa de pesquisas e desenvolvimentos da Organização das Nações Unidas - ONU); 'Food and Agriculture Organization' - FAO (programa de organizações de esforços de controle da produção alimentar mundial e de combate à fome, vinculado a ONU).

\section{Consumidores de alimentos sustentáveis $\mathbf{x}$ consumidores de alimentos industrializados}

Segundo os órgãos internacionais de controle e de produção de alimento mundial, Ciheam et al. (2015), existem várias discussões sobre os padrões propostos de consumo alimentar, que visam transformar este consumo sustentável para o mundo, discussões estas cada vez mais aprofundadas. De acordo com Ciheam et al. (2015), a globalização forçou mudanças em técnicas alimentares e em culturas agroalimentares, em todo planeta Terra, neste sentido houve um aumento da produção de alimentos em massa, principalmente para suprir o mercado das grandes zonas urbanas, entretanto, também, proporcionou uma construção rica e complexa das diversas dietas, reflexo este encontrado nas dietas mediterrâneas transformadas pela globalização; que como relatado no trabalho estão dentro dos tipos de consumidores que buscam uma alimentação sustentável, que se contrapõem, muitas vezes , ao processo de alimentação industrializada.

$\mathrm{Na}$ atualidade as civilizações passam por constantes transições nutricionais, assim: "a transição nutricional ocorre em conjunto com a transição epidemiológica das doenças crônicas e tem sérias implicações em termos de saúde pública, crescimento econômico e política de nutrição" (CHIEAM et al., 2015). Contrapondo a produção em massa dos alimentos industrializados, aponta Ciheam et al. (2015):

Uma boa governança do sistema alimentar é crucial. Surge, cada vez mais, as decisões sobre quem produz os alimentos, que alimentos são produzidos, quando, onde e como esses alimentos são produzidos e quem come, estes sendo produzidos por quem administra um 
pequeno número empresas de alimentos dominantes. As decisões de políticas públicas impactam o sistema alimentar e muitas vezes facilitam que as mudanças estruturais continuem sendo feitas em nível local, regional, nacional e níveis internacionais de governo. Enquanto isso, agricultores, consumidores, formuladores de políticas e comunidades estão tentando lidar com os impactos que a crescente consolidação e concentração, que está ocorrendo em todo o sistema alimentar. (CHIEAM et al., 2015)

Os grandes grupos das produções de alimentos aumentam e com isto a concentração do poder do Capital dessas empresas impedem a diversidade das produções de alimentações sustentáveis indiretamente, assim, não sobra grandes espaços geopolíticos mundiais para o apoio na produção de alimentos saudáveis e sustentáveis.

Segundo Guerra et al. (2017), as ações de consumir e de se alimentar é indissociável da vida humana, contudo é implantado no mercado global alimentar uma cultura do consumismo que difere do ato de consumir; no caso dos brasileiros, exemplo de país onde as crenças e as regras são ditadas pelas mídias, se nota o consumo intenso de carne. O fato citado remete ao consumo alimentar insustentável das sociedades modernas; estabelecido pelo consumismo midiático, conceito de estímulos ao consumo exagerado para suprir os anseios pessoais dos indivíduos sociais, por sua vez, incentivados pelos meios de comunicações. (GUERRA et al., 2017). Apesar de existirem as campanhas de conscientização alimentar, se deduz que o mercado de alimentação sustentável, perde, em grande parte, para o mercado dos alimentos industrializados, sobretudo aqueles que estimulam o consumo exacerbado da proteína animal.

\section{Consumismo alimentar e o consumo consciente atual}

O consumismo no mercado de alimentos como cultura da globalização está consolidado e representa uma grande força no capitalismo, isto já é consenso da população mundial atual. De acordo com Maggio et al. (2015), a agricultura utiliza cerca de $70 \%$ de toda água doce consumida pelos seres humanos, aonde alguns países já experimentam uma redução significativa dessa água, devido os lençóis freáticos em diminuições trágicas, de suas reservas naturais de água doce.

O que se reflete é que indiretamente, as grandes empresas de consumo alimentar industrializado, ao buscarem, cada vez mais lucros, realizam ou estimulam a retirada intensa de recursos naturais, como maior exemplo a redução da água doce, através das grandes produções da agricultura, para sustentar o consumismo em seus grandes mercados de alimentos, urbanizados e industrializados.

Segundo as pesquisas da União Europeia já citadas, Maggio et al. (2015), no cenário mundial, é previsto um colapso na oferta e na demanda por comida até 2030, além da espera das futuras mudanças climáticas, que resultará no deslocamento anual de 12 milhões de pessoas em todo o globo, enquanto isto as mudanças alimentares serão um fator decisivo para todo o mercado de produção de alimentos, sobretudo na agricultura. $\mathrm{O}$ que esperar do consumo de alimentos e dos consumidores conscientes? $\mathrm{O}$ presente trabalho deduz o motivo da formação dos novos consumidores conscientes e os seus tipos de dietas, por causa das preocupações com os vários problemas socioambientais que a humanidade vivencia e vivenciará mais intensamente nos próximos anos. 
Sobre as preocupações do mercado de consumo alimentar e seus principais investimentos, pelo segmento dos consumidores conscientes, aponta-se:

\begin{abstract}
Um foco contínuo na resiliência humana nas regiões com maior insegurança alimentar do mundo e investimentos crescentes que visam atender as deficiências nutricionais em crianças menores de cinco anos, fundamental para a redução das principais inseguranças alimentares em todo o mundo. Em acesso à água potável para populações rurais e urbanas, com ampliação nas suas garantias (problemas relacionados degradação da qualidade da água, de acesso, de resíduos e de desequilíbrio regional). Nos peixes e em seus derivados, estes que têm papel-chave na melhoria do componente proteico das dietas, uma aplicação de uma piscicultura sustentável e efetiva nos sistemas de gestão de pescas, necessário assegurar a sustentabilidade na exploração de recursos naturais renováveis, nos oceanos e às águas interiores, assim como, seus peixes, suas bivalves e suas algas. Assim a pesca e a aquicultura estão contribuindo substancialmente para fornecendo alimentos de alto valor nutritivo para dietas saudáveis. (MAGGIO et al., 2015)
\end{abstract}

As pesquisas até aqui demonstradas, mostram uma possível futura crescente dos consumidores conscientes, que buscam as dietas da suficiência alimentar, semelhante aos adeptos das dietas sustentáveis, esses são adeptos aos alimentos mais saudáveis para o organismo humano, contudo suas preocupações são mais relevantes com a preservação dos ecossistemas da terra, que são fontes naturais da alimentação humana. De forma elucidativa e posteriormente ilustrativa (imagem a baixo), a visão da busca de uma segurança mundial na alimentação da União Europeia, representa em maior parte, o perfil de consumo alimentar dos indivíduos adeptos as dietas da suficiência.

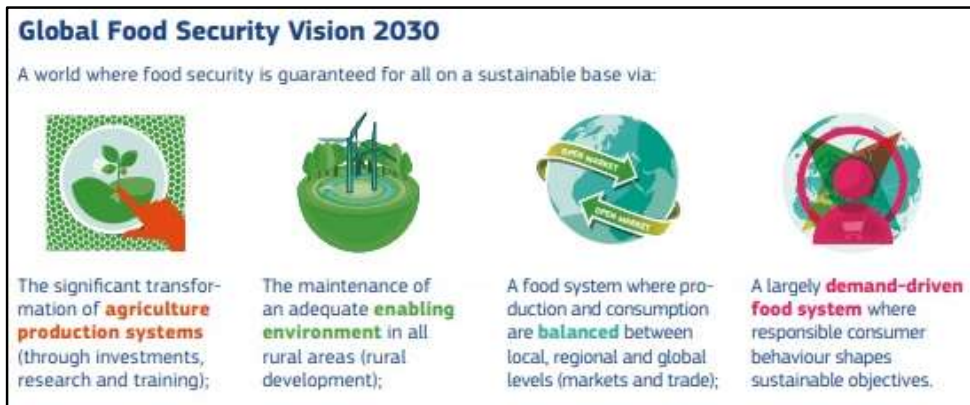

Figura 1: Perfil de consumo alimentar. Fonte: Maggio et al. (2015).

Assim, como a visão da União Europeia demonstrada, os consumidores alimentares da suficiência prezam pela construção de uma produção alimentar adequada a preservação e a revitalização dos recursos naturais existentes, que estão em diminuições significativas.

\title{
Consumidores sem entendimento da sua alimentação e os inovadores em sua alimentação
}

Segundo as pesquisas, Ciheam et al. (2015), O aumento populacional e a intensa urbanização, além da globalização, em toda Terra, afetaram profundamente os ecossistemas e as dietas humanas; As problemáticas são preocupantes, estas: 1) a produção e o processamento de alimentos não são sustentáveis, devido a devastação da natureza e a não preservação de seus recursos naturais, uma parcela deste fato ocasionado pelas indústrias alimentícias; 2) as situações degradantes de boa parte das zonas rurais; 3 ) consumo excessivo de alimentos pelos seres humanos.

As modernidades trazem diversos avanços, desde tecnológicos até socioeconômicos, entretanto, de acordo com Garcia (2003), os ideais de progresso a qualquer custo afetam a produção de alimentos, que no 
mercado capitalista exige rapidez e praticidade, desconsiderando aspectos relevantes da segurança alimentar, como o incentivo ao consumo de uma alimentação saudável. Neste sentido, os indivíduos são influenciados pelas ideologias das publicidades dos produtos alimentícios industrializados.

Contrapondo as buscas das dietas ideais, segundo Garcia (2003), as dietas tradicionais saudáveis, exemplo as dietas mediterrâneas, representam em seu caráter geral um padrão de pertencimento a uma cultura globalizada, mais que a busca de uma melhoria de saúde. Aponta-se: "Neste caso seria a estilização da dieta mediterrânea que produziria um padrão (pattern) de pertencimento à cultura globalizada. Já não seria a dieta mediterrânea, mas um pattern dela, apropriado para exportação e circulação no mundo globalizado" (GARCIA, 2003).

A partir do processo hipotético-dedutivo se reflete que a falta de conhecimento cientifico alimentar, sobretudo nas muitas informações transmitidas pelas mídis sociais são mal interpretadas ou deixam ideias sobre a alimentação abertas para interpretações erradas, isto faz com que algumas pessoas adotem dietas da moda sem acompanhamento profissional adequado, resultando em diversos prejuízos à saúde, como deficiências nutricionais.

Com as percepções das várias problemáticas alimentares surgem diversos desafios para o setor de produção e de consumo alimentar, de acordo com Moratoya et al. (2013), um dos desafios são estimular as pessoas e as agroindústrias para a produção de alimentos processados que ao mesmo tempo sejam saudáveis e agradáveis para todos os indivíduos sociais.

Entre as inovações e os consumidores criativos e inovadores, de acordo com Moratoya et al. (2013), surgem os nutraceúticos (profissionais que propõem suprimento nutricional através de componentes nutricionais sintéticos), neste sentido ajudam os indivíduos sociais a buscarem melhores qualidades de vida e auxiliam na prevenção de várias doenças. Outra inovação são os adeptos a categoria de alimentos chamados 'nanofood', segundo Moratoya et al. (2013), são os alimentos cultivados e produzidos a partir da utilização de nanotecnologias; em geral são alimentos produzidos com novas tecnologias que proporcionam intensificações nos sabores dos alimentos, melhoras nos seus aspectos nutricionais e a criação de alimentos mais funcionais.

No presente momento do trabalho, surge o início da área da 'nutrigenômica', são as relações dos estudos nutricionais e dos estudos dos genomas, de acordo com Moratoya et al. (2013), que produzem, de forma tecnológica, as mudanças nos genes dos alimentos para mudar as estruturas dos nutrientes, consequentemente, resultando em benefícios nutricionais mais intensos aos seres humanos. Todos os adeptos e incentivadores das inovações alimentares no mercado global de alimentação se encaixam nos consumidores criativos e inovadores, no trabalho categorizado como um tipo de estilo de consumo alimentar.

\section{CONCLUSÕES}

A globalização, dita através da produção em massa de alimentos e do consumo alimentar mundial, de acordo com os padrões alimentares identificados, no presente estudo, configurados em cinco tipos de 
conjunturas de consumidores alimentares, influenciam e são influenciados pelo mercado de consumo alimentar globalizado.

Com a crescente urbanização e industrialização da humanidade, os padrões alimentares mudaram e se encontram em contínuas transformações. É identificado que a produção em massa dos alimentos, principalmente os industrializados e os processados, precisarão de renovações para se tornarem sustentáveis ao mundo atual.

A partir das diminuições significativas dos recursos naturais da Terra, surgem várias propostas de dietas alimentares humanas, associadas a busca do Homem por melhores qualidades de vida. As perspectivas futuras para o setor de consumo de alimentos retratam políticas diversas, que se adaptam as necessidades criadas pelos indivíduos sociais atuais e seus meios de comunicações, estes dois elementos sociais se correlacionam com as políticas internacionais de controle e produção de alimento, assim são formados novos padrões alimentares, consequentemente, estes criam novos estilos de vidas.

\section{REFERÊNCIAS}

ALMEIDA, J. C.; RODRIGUES, T. C.; SILVA, F. M.; AZEVEDO, M. J.. Revisão sistemática de dietas de emagrecimento: papel dos componentes dietéticos. Arq. Bras. Endocrinol Metab., v.53, n.5, p.673-87, 2009.

CIHEAM; FAO. Organização das Nações Unidas para Alimentação e Agricultura. Mediterranean food consumption patterns: diet, environment, society, economy and health. Rome: CIHEAM; Bari: FAO, 2015.

MAGGIO, A.; VAN CRIEKINGE, T.; MALINGREAU, J. P.. Global Food Security 2030: Assessing trends with a view to guiding future EU policies. Luxembourg: Publications Office of the European Union, 2015.

MOOMAW, W.; GRIFFIN, K. T.; KURCZAK, J. L.. The Critical Role of Global Food Consumption Patterns in Achieving Sustainable Food Systems and Food for All. Paris: UNEP, 2012.
MORATOYA, E. E.; CAVALHÃES, G. C.; WENDER, A. E.; ALMEIDA, L. M. M. C.. Mudanças no padrão de consumo alimentar no Brasil e no mundo. Revista de Política Agrícola, v.22, n.1, p.72-84, 2013.

GARCIA, R. W. D.. Reflexos da globalização na cultura alimentar: considerações sobre as mudanças na alimentação urbana. Revista de Nutrição, v.16, n.4, p.483-492, 2003.

GIL, A. C.. Métodos e técnicas de pesquisa social. 6 ed. São Paulo: Atlas, 2008.

GUERRA, C. S.; CARDOSO, F. B. S.. A influência da cultura do consumo na alimentação humana: a (in) sustentabilidade do consumo de proteína animal. In: CONGRESSO INTERNACIONAL DE DIREITO E CONTEMPORANEIDADE, 4. Anais. Santa Maria: UFSM, 2017.

A CBPC - Companhia Brasileira de Produção Científica (CNPJ: 11.221.422/0001-03) detém os direitos materiais desta publicação. Os direitos referem-se à publicação do trabalho em qualquer parte do mundo, incluindo os direitos às renovações, expansões e disseminações da contribuição, bem como outros direitos subsidiários. Todos os trabalhos publicados eletronicamente poderão posteriormente ser publicados em coletâneas impressas sob coordenação da Sapientiae Publishing, da Companhia Brasileira de Produção Científica e seus parceiros autorizados. Os (as) autores (as) preservam os direitos autorais, mas não têm permissão para a publicação da contribuição em outro meio, impresso ou digital, em português ou em tradução. 